\title{
Reproductive outcomes in men with karyotype abnormalities: Case report and review of the literature
}

\author{
Taylor P. Kohn, MPhil; ${ }^{*}$ Raul Clavijo, MD; ${ }^{\dagger}$ Ranjith Ramasamy, MD;* Tariq Hakky, MD;* \\ Aravind Candrashekar, MD, MBA, " Dolores J. Lamb, PhD, HCLD,; Larry I. Lipshultz, MD*
}

*Department of Urology, Baylor College of Medicine, Houston, TX; 'Department of Urology, University of California, Los Angeles, CA

Cite as: Can Urol Assoc J 2015;9(9-10):E667-70. http://dx.doi.org/10.5489/cuaj.2902 Published online September 9, 2015.

\section{Abstract}

Reciprocal translocations of autosomal chromosomes are present in about 1/625 men, yet often there are no symptoms except primary infertility. Abnormal segregation during meiosis often produces sperm and subsequent embryos with unbalanced translocations that often ultimately result in spontaneous abortions. We report on a 37-year-old man and his 39-year-old wife who complained of primary infertility. Previous in vitro fertilization (IVF) had resulted in pregnancy, but two spontaneous abortions. Upon chromosomal testing, the man was diagnosed with a reciprocal translocation and his wife was diagnosed with mosaic Turner's syndrome. Through testicular sperm extraction (TESE) and IVF with preimplantation genetic screening (PGS), they succeeded in having two healthy children. Since men with different karyotype abnormalities can have male infertility, we reviewed the literature and summarized the reproductive outcomes for men with both autosome and sex chromosomal karyotype abnormalities.

\section{Case report}

A 37-year-old man and his 39-year-old wife presented due to a complaint of primary infertility. They had been trying to conceive for 12 years. Two years previously, the patient had undergone bilateral testicular biopsies with successful sperm retrieval and conception. Unfortunately, the couple experienced recurrent pregnancy loss (both during first trimester) following two cycles of in vitro fertilization (IVF) and intracytoplasmic sperm injection (ICSI) without genetic testing. On examination, the man had bilaterally descended testis with a right testicular size of $18 \mathrm{cc}$ and a left testicle size of 16 cc. No varicocele was palpable; the vas deferens and epididymis were palpable bilaterally. Testosterone levels were $241 \mathrm{ng} / \mathrm{dL}$, follicle stimulating hormone levels $7 \mathrm{mIU} / \mathrm{mL}$, and luteinizing hormone levels were $5 \mathrm{mIU} / \mathrm{mL}$. Semen analysis revealed no sperm. Based on the evalua- tion and previous operation at the outside institution, the patient was diagnosed with azoospermia likely secondary to epididymal obstruction.

The couple was sent for genetic evaluation due to recurrent pregnancy loss. Genetic evaluation showed that the husband had a reciprocal translocation involving chromosomes 4 and 8 [46,XY;t(4;8)(q31.1;q22.3)]. In addition, his wife had $45, X / 46, X X$ mosaicism. The patient underwent a successful right testicular sperm extraction (TESE) with IVF/ICSI combined with pre-implantation genetic screening (PGS). The result was a successful live birth. Two years later, a repeat TESE was performed for another IVF/ICSI/PGS cycle, and the couple had a second healthy child.

\section{Discussion}

The prevalence of reciprocal translocations in the general population is about $1 / 625 .{ }^{1}$ This prevalence is greater in infertile couples (1/166), in couples who have failed to achieve a pregnancy after $>10$ total embryos transferred for IVF (1/31), and in couples who have experienced $\geq 3$ consecutive first-trimester spontaneous abortions $(1 / 11){ }^{2}$ Studies have shown that for couples in whom the male has a reciprocal translocation, $75 \%$ of natural pregnancies will result in a spontaneous abortion, with a live birth rate of only $4.9 \% .^{3,4}$ Increased frequency of male infertility in men with reciprocal translocations is due to abnormal meiosis during spermatogenesis. ${ }^{5}$ Abnormal segregation during meiosis can result in sperm with unbalanced translocations (i.e., chromosomal duplications and deletions). ${ }^{6}$ Embryos with unbalanced translocations are at a high risk of spontaneous abortion, stillbirth, or neonatal anomalies, and only $11.5 \%$ experience births of healthy infants. This explains why couples with a reciprocal translocation have significant problems conceiving and carrying to term. ${ }^{7}$

In recent years, preimplantation genetic screening (PGS) has significantly improved the frequency of healthy births in couples with genetic abnormalities. Screening the embryo 
Kohn et al.

Table 1. Summary of literature pertaining to sperm retrieval in patients with autosomal and sex chromosome abnormalities

\begin{tabular}{|c|c|c|c|c|c|c|c|c|}
\hline Study & Age & Year & Karyotype abnormality & $\begin{array}{l}\text { No. } \\
\text { cases }\end{array}$ & Semen analysis & $\begin{array}{c}\text { Sperm } \\
\text { retrieval }\end{array}$ & $\begin{array}{l}\text { Assisted } \\
\text { Fertility }\end{array}$ & Pregnancy \\
\hline \multicolumn{9}{|c|}{ Autosomal chromosome abnormality } \\
\hline Current case & 42 & 2015 & $46, X Y, t(4 ; 8)(q 31.1 ; q 22.3)$ & 1 & Azoospermia & Yes & Yes & Yes \\
\hline Ananthapur et al. ${ }^{8}$ & 34 & 2013 & $46, X Y, t(2 ; 11)(p 14 ; q 21)$ & 1 & Oligospermia & No & No & Yes (3) \\
\hline Almeida et al. ${ }^{9}$ & 31 & 2012 & $46, X Y, t(2 ; 2)(p 25.1 ; q 23)$ & 1 & Oligoasthenozoospermia & No & No & Yes \\
\hline Motoyama et al. ${ }^{10}$ & 28 & 2011 & $46, X Y, t(10 ; 21)(q 11.2 ; p 11.2)$ & 1 & Oligoasthenozoospermia & Yes & Yes & Yes \\
\hline Joly-Helas et al. ${ }^{11}$ & 35 & 2007 & $46, X Y, t(4 ; 11)(q 34 ; q 13.5)$ & 1 & Oligospermia & No & Yes & Yes \\
\hline Drouineaud et al..$^{12}$ & 34 & 2003 & $45, X Y, \operatorname{der}(13 ; 14),(q 10 ; q 10)$ & 1 & Azoospermia & Yes & Yes & Yes \\
\hline Cai et al. ${ }^{13}$ & 35 & 2000 & $\begin{array}{c}46, X Y, t(7 ; 9)(q 22 ; p 24), \text { ins }(8 ; 7) \\
(q 21.2 ; q 22 q 32) . i s h \operatorname{der}(9) \\
(w c p 7+) ; i n s(8 ; 7) \\
(w c p 8+, w c p 7+)\end{array}$ & 1 & Oligospermia & No & No & Yes \\
\hline Belin et al. ${ }^{14}$ & NA & 1999 & $46, X Y, t(20 ; 22)(q 12.0 ; q 11.21)$ & 1 & Oligospermia & NA & Yes & Yes \\
\hline Meschede et al. ${ }^{15}$ & NA & 1997 & $46, X Y, t(1 ; 9)(q 44 ; p 11.2)$ & 1 & Oligospermia & NA & Yes & Yes \\
\hline Veld et al. ${ }^{16}$ & $41, N A$ & 1997 & $\begin{array}{c}\text { 45,XY,der(13;13)(q10; } \\
q 10) / 46, X Y, t(13 ; 13)(p 10 ; p 10), \\
\operatorname{der}(13 p ; 13 p) \\
\text { AND } \\
45, X Y, \operatorname{der}(13 ; 14)(q 10 ; q 10)\end{array}$ & 2 & Oligospermia & No & Yes & Yes \\
\hline \multicolumn{9}{|c|}{ Sex chromosome abnormality } \\
\hline $\begin{array}{l}\text { Flannigan RK et } \\
\text { al. }^{17}\end{array}$ & 27 & 2014 & $45, X / 46, X Y$ & 1 & Azoospermia & Yes & Yes & No \\
\hline Abdel-Razic et al. ${ }^{18}$ & $23-40$ & 2011 & $47, X Y Y$ & 9 & $\begin{array}{l}\text { Oligospermia (7) } \\
\text { Azoospermia (2) }\end{array}$ & No & Yes (2) & Yes (1) \\
\hline Kilic et al. ${ }^{19}$ & $25-32$ & 2010 & $45, X / 46, X Y$ & 3 & $\begin{array}{l}\text { Azoospermia (2) } \\
\text { Oligospermia (1) }\end{array}$ & Yes (2) & Yes (1) & No \\
\hline Spinner et al. ${ }^{20}$ & NA & 2008 & $46, \mathrm{Xr}(\mathrm{Y})$ & 1 & Oligospermia & Yes & Yes & Yes \\
\hline Sugawara et al. ${ }^{21}$ & 27 & 2005 & $46, X X / 46, X Y$ & 1 & Azoospermia & Yes & Yes & Yes \\
\hline
\end{tabular}

prior to implantation assures that only those embryos with appropriate numbers of chromosomes are implanted. For couples in whom one or more partners have a reciprocal translocation, preimplantation genetic diagnosis (PGD) reduced the frequency of spontaneous abortions to $12.5 \%$ and increased the live birth rate to more than $80 \%{ }^{3,4}$

Due to the complexity of translocations and the high incidence of chromosomal aneuploidy with similar phenotypes, a review of the existing literature was critical (Table 1 ). Most patients with chromosomal abnormalities, with the notable exception of patients with $45, X / 46, X Y$ including those with complex chromosomal rearrangements, can still achieve pregnancies. This literature review reveals that sperm retrieval appears necessary in the presence of many sex chromosomal abnormalities, whereas patients with autosomal chromosomal abnormalities are often oligospermic and thus do not require testicular sperm extraction. Further, it appears that for patients with sex chromosomal abnormalities, including those with Kleinfelter syndrome, sperm retrieval combined with assisted fertility treatment offers a good prognosis for pregnancy.

While in this case report the IVF/ICSI attempts resulted with two healthy, live births, there is an ethical concern that an abnormal karyotype might be passed onto the prodigy. In countries where the law does not preclude assisted reproductive techniques for couples with a balanced chromosomal translocations, the couple ought to be referred to genetic counselling and be advised that an abnormal karyotype might be passed onto the prodigy before starting assisted reproductive techniques.

\section{Conclusion}

We report a successful pregnancy using TESE and IVF/ICSI in a couple in whom both individuals had abnormal karyotypes. The use of sperm extraction techniques and assisted fertility treatments, including pre-implantation genetic screening, dramatically helped these patients with chromosomal abnormalities achieve viable pregnancies. We expect genetic defects in men with infertility will be diagnosed with greater precision (beyond translocation) since molecular diagnostics, such as next-generation sequencing and microarray-based comparative genomic hybridization (array-CGH) analysis, have now been incorporated into clinical labs. 


\begin{tabular}{|c|c|c|c|c|c|c|c|c|}
\hline Study & Age & Year & Karyotype abnormality & $\begin{array}{l}\text { No. } \\
\text { cases }\end{array}$ & Semen analysis & $\begin{array}{l}\text { Sperm } \\
\text { retrieval }\end{array}$ & $\begin{array}{l}\text { Assisted } \\
\text { Fertility }\end{array}$ & Pregnancy \\
\hline \multicolumn{9}{|c|}{ Kleinfelter syndrome } \\
\hline Sabbaghian et al. ${ }^{22}$ & $\begin{array}{c}32 \\
\text { (mean) }\end{array}$ & 2014 & $47, X X Y$ & 134 & Azoospermia & Yes (38) & Yes (18) & Yes (4) \\
\hline Greco et al. ${ }^{23}$ & $\begin{array}{c}35 \\
\text { (mean) }\end{array}$ & 2013 & $47, X X Y$ & 38 & Azoospermia & Yes (15) & Yes (11) & Yes (11) \\
\hline Vicdan et al. ${ }^{24}$ & 35 & 2011 & $47, X X Y$ & 1 & Azoospermia & Yes & Yes & Yes \\
\hline Ramasamy et al..$^{25}$ & $\begin{array}{c}33 \\
\text { (mean) }\end{array}$ & 2009 & $47, X X Y$ & 68 & Azoospermia & Yes (45) & Yes (45) & Yes (33) \\
\hline Yarali et al. ${ }^{26}$ & $\begin{array}{c}32 \\
\text { (mean) }\end{array}$ & 2009 & $47, X X Y$ & 33 & Azoospermia & Yes (22) & Yes (22) & Yes (7) \\
\hline Kyono et al. ${ }^{27}$ & $\begin{array}{c}30 \\
\text { (mean 2) } \\
38 \\
\text { (mean 1) }\end{array}$ & 2007 & $47, X X Y$ & 17 & Azoospermia & Yes (6) & Yes (6) & Yes (5) \\
\hline Koga et al. ${ }^{28}$ & $\begin{array}{c}36 \\
\text { (mean) }\end{array}$ & 2007 & $47, X X Y$ & 26 & Azoospermia & Yes (13) & Yes (13) & Yes (3) \\
\hline Schiff et al. ${ }^{29}$ & $24-52$ & 2005 & $\begin{array}{l}\text { 47,XXY (39) AND 46, } \\
\text { XX/47,XXY(3) }\end{array}$ & 42 & Azoospermia & Yes (29) & Yes (29) & Yes (18) \\
\hline Okada et al..$^{30}$ & $25-43$ & 2005 & $47, X X Y$ & 51 & Azoospermia & Yes (26) & Yes (26) & Yes (12) \\
\hline Seo et al. ${ }^{31}$ & $26-42$ & 2004 & $\begin{array}{c}\text { 47,XXY (25) AND 46, } \\
\text { XY/47,XXY(11) }\end{array}$ & 36 & Azoospermia & Yes (10) & Yes (10) & Yes (4) \\
\hline Vernaeve et al. ${ }^{32}$ & $\begin{array}{c}29.5 \\
\text { (mean) }\end{array}$ & 2004 & $47, X X Y$ & 50 & Azoospermia & Yes (24) & NA & NA \\
\hline Westlander et al. ${ }^{33}$ & $\begin{array}{c}33 \\
\text { (mean) }\end{array}$ & 2003 & $47, X X Y$ & 18 & Azoospermia & Yes (5) & Yes (5) & Yes (2) \\
\hline Yamamoto et al..$^{34}$ & NA & 2002 & $47, X X Y$ & 24 & Azoospermia & Yes (12) & Yes (12) & Yes (4) \\
\hline Friedler et al..$^{35}$ & $\begin{array}{c}28.7 \\
\text { (mean) }\end{array}$ & 2001 & $47, X X Y$ & 12 & Azoospermia & Yes (5) & Yes (5) & Yes (5) \\
\hline Cruger et al. ${ }^{36}$ & 28 & 2001 & $47, X X Y$ & 1 & Oligospermia & No & Yes & Yes \\
\hline Poulakis et al. ${ }^{37}$ & 33,35 & 2001 & $47, X X Y$ & 2 & Azoospermia & Yes & Yes & Yes \\
\hline Levron et al. ${ }^{38}$ & NA & 2000 & $47, X X Y$ & 20 & Azoospermia & Yes (8) & Yes (8) & Yes (4) \\
\hline Ron-El et al. ${ }^{39}$ & 31 & 2000 & $47, X X Y$ & 1 & Azoospermia & Yes & Yes & Yes \\
\hline Nodar et al. ${ }^{40}$ & 39 & 1999 & $47, X X Y$ & 1 & Azoospermia & Yes & Yes & Yes \\
\hline Tourmaye et al. ${ }^{41}$ & NA & 1997 & $47, X X Y$ & 15 & Azoospermia & Yes (8) & Yes (7) & Yes (2) \\
\hline
\end{tabular}

Competing interests: The authors all declare no competing financial or personal interests.

This paper has been peer-reviewed.

\section{References}

1. Van Dyke DL, Weiss L, Roberson JR, et al. The frequency and mutation rate of balanced autosomal rearrangements in man estimated from prenatal genetic studies for advanced maternal age. Am J Hum Genet 1983;35:301-8.

2. Stern C, Pertile $\mathrm{M}$, Norris $\mathrm{H}$, et al. Chromosome translocations in couples with in-vitro fertilization implantation failure. Hum Reprod 1999;14:2097-101. http://dx.doi.org/10.1093/humrep/14.8.2097

3. Keymolen K, Staessen C, Verpoest W, et al. Preimplantation genetic diagnosis in female and male carriers of reciprocal translocations: clinical outcome until delivery of 312 cycles. Eur J Hum Genet 2012;20:37680. http://dx.doi.org/10.1038/ejhg.2011.208

4. Munne $S$, Sandalinas M, Escudero T, et al. Outcome of preimplantation genetic diagnosis of translocations. Fertil Steril 2000;73:1209-18. http://dx.doi.org/10.1016/S0015-0282(00)00495-7
5. Lipshultz LI, Howards SS and Niederberger CS. Infertility in the male. 4th ed. New York,NY: Cambridge University Press; 2009. http://dx.doi.org/10.1017/CB09780511635656

6. Brandriff $B$, Gordon L, Ashworth LK, et al. Cytogenetics of human sperm: Meiotic segregation in two translocation carriers. Am J Hum Genet 1986;38:197-208.

7. Verlinsky Y, Tur-Kaspa I, Cieslak J, et al. Preimplantation testing for chromosomal disorders improves reproductive outcome of poor-prognosis patients. Reprod Biomed Online 2005;11:219-25. http://dx.doi. org/10.1016/S1472-6483(10)60961-3

8. Ananthapur V, Avvari S, Veena K, et al. Non-Robertsonian translocation $\dagger(2 ; 11)$ is associated with infertility in an oligospermic man. Andrologia, 2014;46:453-5. http://dx.doi.org/10.1111/and.12120

9. Almeida C, Dória $S$, Moreira $M$, et al. Normal sperm in a 2;2 homologous male translocation carrier. J Assist Reprod Genet 2012;29:665-8. http://dx.doi.org/10.1007/s10815-012-9770-6

10. Motoyama M, Takahashi K, Ogawa $S$, et al. Chromosome analysis by spectral karyotyping of spermatozoa from an oligoasthenozoospermic carrier of a 10; 21 reciprocal translocation. Hum Cell 2011;24:146-9. http://dx.doi.org/10.1007/s13577-011-0035-y

11. Joly-Helas G, de La Rochebrochard C, Mousset-Siméon N, et al. Complex chromosomal rearrangement and intracytoplasmic sperm injection: A case report. Hum Reprod 2007;22:1292-7. http://dx.doi. org/10.1093/humrep/del507

12. Drouineaud $V$, Sagot $P$, Faivre $L$, et al. Birth after intracytoplasmic injection of epididymal sperm from a man with congenital bilateral absence of the vas deferens who had a robertsonian translocation. Fertil Steril 2003;79(Suppl 3);1649-51. http://dx.doi.org/10.1016/S0015-0282(03)00341-8 
Kohn et al.

13. Cai T, Yu P, Tagle DA, et al. A de novo complex chromosomal rearrangement with a translocation 7;9 and $8 q$ insertion in a male carrier with no infertility: Case report. Hum Reprod 2001;16:59-62. http:// dx.doi.org/10.1093/humrep/16.1.59

14. Belin V, Farhat $M$, Monset-Couchard $M$. Intracytoplasmic sperm injection pregnancy with trisomy 20p and monosomy 22q in a newborn resulting from a balanced paternal translocation. Neonatology 1999;75:398401. http://dx.doi.org/10.1159/000014121

15. Meschede D, Louwen F, Eiben B, et al. Intracytoplasmic sperm injection pregnancy with fetal trisomy 9p resulting from a balanced paternal translocation. Hum Reprod 1997;12:1913-4. http://dx.doi. org/10.1093/humrep/12.9.1913

16. Veld PA, Weber RF, Los FJ, et al. Two cases of Robertsonian translocations in oligozoospermic males and their consequences for pregnancies induced by intracytoplasmic sperm injection. Hum Reprod 1997;12:1642-4. http://dx.doi.org/10.1093/humrep/12.8.1642

17. Flannigan RK, Chow V, Ma S, et al. $45, X / 46, X Y$ mixed gonadal dysgenesis: A case of successful sperm extraction. Can Urol Assoc J 2014;8:E108-E10. http://dx.doi.org/10.5489/cuaj.1574

18. Abdel-Razic MM, Abdel-Hamid IA, ElSobky ES. Nonmosaic 47,XYY syndrome presenting with male infertility: Case series. Andrologia 2012;44:200-04. http://dx.doi.org/10.1111/i.1439-0272.2010.01129.x

19. Kilic $S$, Yukse B, Tasdemir N, et al. Assisted reproductive treatment applications in men with normal phenotype but 45,x/46,xy mosaic karyotype: Clinical and genetic perspectives. Taiwan J Obstet Gyne 2010;49:199-202. http://dx.doi.org/10.1016/S1028-4559(10)60042-3

20. Spinner NB, Saitta SC, Delaney DP, et al. Intracytoplasmic sperm injection (ICSI) with transmission of a ring $(Y)$ chromosome and ovotesticular disorder of sex development in offspring. Am J Med Genet A 2008;146A:1828-31. http://dx.doi.org/10.1002/aimg.a.32358

21. Sugawara $\mathrm{N}$, Tokunaga $\mathrm{Y}$, Maeda $\mathrm{M}$, et al. A successful pregnancy outcome using frozen testicular sperm from a chimeric infertile male with a 46, XX/46, XY karyotype: Case report. Hum Reprod 2005;20:147-8. http://dx.doi.org/10.1093/humrep/deh587

22. Sabbaghian M, Modarresi T, Hosseinifar $\mathrm{H}$, et al. Comparison of sperm retrieval and intracytoplasmic sperm injection outcome in patients with and without klinefelter syndrome. Urology 2014;83:107-10. http://dx.doi.org/10.1016/i.urology.2013.09.021

23. Greco E, Scarselli F, Minasi MG, et al. Birth of 16 healthy children after ICSI in cases of nonmosaic Klinefelter syndrome. Hum Reprod 2013;28:1155-60. http://dx.doi.org/10.1093/humrep/det046

24. Vicdan K, Akarsu C, Vicdan A, et al. Birth of a healthy boy using fresh testicular sperm in a patient with Klinefelter syndrome combined with Kartagener syndrome. Fertil Steril 2011;96:577-9. http://dx.doi. org/10.1016/i.fertnstert.2011.06.011

25. Ramasamy R, Ricci JA, Palermo GD, et al. Successful fertility treatment for Klinefelter's syndrome. J Urology 2009;182:1108-13. http://dx.doi.org/10.1016/i.jur.2009.05.019

26. Yarali H, Polat $M$, Bozdag $G$, et al. TESE-ICSI in patients with non-mosaic Klinefelter syndrome: A comparative study. Reprod Biomed Online 2009;18:756-60. http://dx.doi.org/10.1016/S14726483(10)60023-5

27. Kyono $\mathrm{K}$, Uto $\mathrm{H}$, Nakajo $\mathrm{Y}$, et al. Seven pregnancies and deliveries from non-mosaic Klinefelter syndrome patients using fresh and frozen testicular sperm. J Assist Reprod Genet 2007;24:47-51. http://dx.doi. org/10.1007/s10815-006-9079-4

28. Koga $M$, Tsü̈mura $A$, Takeyama $M$, et al. Clinical comparison of successful and failed microdissection testicular sperm extraction in patients with nonmosaic Klinefelter syndrome. Urology 2007;70:341-5. http://dx.doi.org/10.1016/j.urology.2007.03.056
29. Schiff JD, Palermo GD, Veeck $L L$, et al. Success of testicular sperm injection and intracyłoplasmic sperm injection in men with Klinefelter syndrome. J Clin Endocr Metab 2005;90:6263-7. http://dx.doi. org/10.1210/ic.2004-2322

30. Okada H, Goda K, Yamamoto Y, et al. Age as a limiting factor for successful sperm retrieval in patients with nonmosaic Klinefelter's syndrome. Fertil Steril 2005;84:1662-4. http://dx.doi.org/10.1016/i. fertnstert.2005.05.053

31. Seo JT, Park YS, Lee JS. Successful testicular sperm extraction in Korean Klinefelter syndrome. Urology 2004;64:1208-11. http://dx.doi.org/10.1016/i.urology.2004.07.013

32. Vernaeve V, Staessen C, Verheyen G, et al. Can biological or clinical parameters predict testicular sperm recovery in 47,XXY Klinefelter's syndrome patients? Hum Reprod 2004;19:1135-9. http://dx.doi. org/10.1093/humrep/deh253

33. Westlander G, Ekerhovd E, Bergh C. Low levels of serum inhibin B do not exclude successful sperm recovery in men with nonmosaic Klinefelter syndrome. Fertil Steril 2003;79(Suppl 3);1680-2. http:// dx.doi.org/10.1016/S0015-0282(03)00403-5

34. Yamamoto Y, Sofikitis N, Mio Y, et al. Morphometric and cytogenetic characteristics of testicular germ cells and sertoli cell secretory function in men with non-mosaic Klinefelter's syndrome. Hum Reprod 2002;17:886-96. http://dx.doi.org/10.1093/humrep/17.4.886

35. Friedler S, Raziel A, Strassburger D, et al. Outcome of ICSI using fresh and cryopreserved-thawed testicular spermatozoa in patients with non-mosaic Klinefelter's syndrome. Hum Reprod 2001;16:2616-20. http:// dx.doi.org/10.1093/humrep/16.12.2616

36. Crüger D, Toft B, Agerholm I, et al. Birth of a healthy girl after ICSI with ejaculated spermatozoa from a man with non-mosaic Klinefelter's syndrome: Case report. Hum Reprod 2001;16:1909-11. http:// dx.doi.org/10.1093/humrep/16.9.1909

37. Poulakis V, Witzsch U, Diehl W, et al. Birth of two infants with normal karyotype after intracytoplasmic injection of sperm obtained by testicular extraction from two men with nonmosaic Klinefelter's syndrome. Fertil Steril 2001;76:1060-2.

38. Levron J, Aviram-Goldring A, Madgar I, et al. Sperm chromosome analysis and outcome of IVF in patients with non-mosaic Klinefelter's syndrome. Fertil Steril 2000;74:925-9. http://dx.doi.org/10.1016/ S0015-0282(00)01556-9

39. Ron-EI R, Strassburger D, Gelman-Kohan S, et al. A 47,XXY fetus conceived after ICSI of spermatozoa from a patient with non-mosaic Klinefelter's syndrome: Case report. Hum Reprod 2000;15:1804-06. http://dx.doi.org/10.1093/humrep/15.8.1804

40. Nodar F, De Vincentiis S, Olmedo SB, et al. Birth of twin males with normal karyotype after intracyłoplasmic sperm injection with use of testicular spermatozoa from a nonmosaic patient with Klinefelter's syndrome. Fertil Steril 1999;71:1149-52. http://dx.doi.org/10.1016/S0015-0282(99)00151-X

41. Tournaye $H$, Camus $M$, Vandervorst $M$, et al. Surgical sperm retrieval for intracytoplasmic sperm injection. Int J Androl 1997;20(Suppl 3):69-73.

Correspondence: Dr. Larry Lipshultz, 6624 Fannin St., Suite 1700, Houston, TX 77030; larry|@bcm.edu 